\title{
FNCLCC Sarcoma Grade 1
}

National Cancer Institute

\section{Source}

National Cancer Institute. FNCLCC Sarcoma Grade 1. NCI Thesaurus. Code C9024.

A sarcoma with a total score of 2 or 3 according to the FNCLCC guidelines. 